\title{
Temperature-dependent displacement cross section of graphene and its impurities: measuring the
} carbon adatom migration barrier

Andreas Postl ${ }^{1}$, Jacob Madsen ${ }^{2}$, Pit Pascal Patrick Hilgert ${ }^{2}$, Makoto Tokoro Schreiber ${ }^{3}$, Jani Kotakoski ${ }^{4}$ and Toma Susi ${ }^{5}$

${ }^{1}$ Faculty of Physics \& VDS Physics, University of Vienna, Vienna, Austria, Vienna, Wien, Austria, ${ }^{2}$ Faculty of Physics, University of Vienna, Vienna, Austria, United States, ${ }^{3}$ Okinawa Institute of Science and Technology Graduate University (OIST), Japan, Japan, ${ }^{4}$ Faculty of Physics, University of Vienna, Vienna, Austria, Vienna, Wien, Austria, ${ }^{5}$ Faculty of Physics, University of Vienna, Vienna, Austria, Vienna, Austria

Surface diffusion is crucial for many physical and chemical processes, including epitaxial growth of crystals and heterogeneous catalysis. Although the phenomenon is common [1] and theoretically understood, measuring adatom migration barriers on 2D materials remains a daunting challenge. We are able to estimate the carbon adatom migration barrier on freestanding monolayer graphene, which has theoretically been predicted to be in the range of $350-500 \mathrm{meV}[2,3]$, by quantifying the temperature dependence of its electron knock-on damage.

To measure damage and healing rates as accurately as possible, we use $90 \mathrm{keV}$ electrons and choose the fastest possible time for image acquisition with our aberration-corrected scanning transmission electron microscope. Contrary to expectations, the damage rate decreases with increasing temperature, which is due to the fast healing of vacancies by recombination with diffusing adatoms. By comparing the predicted and observed damage rates at 300-1073 K we find a barrier of $140 \mathrm{meV}$, which is the first measurement reported to date.

We further measured the cross sections of electron-driven processes involving single silicon and phosphorus dopants in graphene at elevated temperatures. To aid in the analysis of our image series, we turned to automated structure recognition based on convolutional neural networks. By utilizing deep learning [4|, we quantified for the first time the direct exchange of carbon neighbors with the dopant in the lattice (so-called bond inversion) [5,6], the replacement of the dopants by carbon adatoms, which has emerged as a hindrance to their manipulation, and knock-on damage of a carbon neighbor, which transforms the sites from threefold to fourfold coordination, as a function of sample temperature.

We gratefully acknowledge funding from the European Research Council (ERC) under the European Unions Horizon 2020 research and innovation program (Grant agreement no. 756277-ATMEN) and the Vienna Doctoral School in Physics.

\section{References}

[1] R. Zan et al., Nano Letters 12 (2021) 3936-3940.

[2] A. V. Krasheninnikov et al., Physical Review B 69 (2004) 073402.

[3] P. O. Lehtinen et al., Physical Review Letters 91 (2003) 017202.

[4] Madsen, J. et al., Microscopy and Microanalysis 25(S2) (2019) 166-167.

[5] M. Tripathi et al., Nano Letters 18 (2018) 5319-5323.

[6] C. Su et al., Science Advances 5 (2019) eaav2252. 